\title{
Pharmacology, Part 1: Introduction to Pharmacology and Pharmacodynamics
}

\author{
Geoffrey M. Currie \\ Faculty of Science, Charles Sturt University, Wagga Wagga, New South Wales, Australia, and Regis University, Boston, Massachusetts
}

CE credit: For CE credit, you can access the test for this article, as well as additional JNMT CE tests, online at https://www.snmmilearningcenter.org. Complete the test online no later than June 2021. Your online test will be scored immediately. You may make 3 attempts to pass the test and must answer $80 \%$ of the questions correctly to receive $1.0 \mathrm{CEH}$ (Continuing Education Hour) credit. SNMMI members will have their CEH credit added to their VOICE transcript automatically; nonmembers will be able to print out a CE certificate upon successfully completing the test. The online test is free to SNMMI members; nonmembers must pay $\$ 15.00$ by credit card when logging onto the website to take the test.

There is an emerging need for greater understanding of pharmacology principles among technical staff. Indeed, the responsibility of dose preparation and administration, under any level of supervision, demands a foundational understanding of pharmacology. This is true for radiopharmaceuticals, contrast media, and pharmaceutical interventions or adjunctive medications. Regulation around the same might suggest a need to embed pharmacology theory in undergraduate education programs, and there is a need to disseminate that same foundational understanding to practicing clinicians. Moreover, pharmacology foundations can provide a key understanding of the principles that underpin quantitative techniques (e.g., pharmacokinetics). This article is the first in a series that aims to enhance the understanding of pharmacologic principles relevant to nuclear medicine. This article will deal with the introductory concepts, terminology, and principles that underpin the concepts to be discussed in the remainder of the series. The second article will build on the pharmacodynamic principles examined in this article with a treatment of pharmacokinetics. Article 3 will outline pharmacology relevant to pharmaceutical interventions and adjunctive medications used in general nuclear medicine, article 4 will cover pharmacology relevant to pharmaceutical interventions and adjunctive medications used in nuclear cardiology, and article 5 will discuss the pharmacology related to contrast media associated with CT and MRI. The final article (6) in the series will examine the pharmacology of drugs associated with the crash cart/emergency trolley.

Key Words: drug safety; dose-response curve; drug action; pharmacodynamics; pharmacology; receptors

J Nucl Med Technol 2018; 46:81-86

DOI: 10.2967/jnmt.117.199588

\footnotetext{
Received Jan. 16, 2018; revision accepted Mar. 12, 2018.

For correspondence or reprints contact: Geoffrey M. Currie, Faculty of Science, Locked Bag 588, Charles Sturt University, Wagga Wagga 2678, Australia.

E-mail: gcurrie@csu.edu.au

Published online Mar. 29, 2018.

COPYRIGHT @ 2018 by the Society of Nuclear Medicine and Molecular Imaging.
}

$\mathbf{P}$ harmacology is the scientific study of the action and effects of drugs on living systems and the interaction of drugs with living systems (1-5). Pharmacology includes the study of prescribed and over-the-counter medications, legal and illicit drugs, natural and synthetic compounds, exogenous (sourced from outside the body) and endogenous (produced inside the body) drugs, and drugs that produce benefit, harm, or both benefit and harm (1-5). Generally speaking, pharmacology is divided into pharmacodynamics and pharmacokinetics (Fig. 1), and these will be described in detail in parts 1 and 2 of this series, respectively. It should, however, be recognized that some texts add the subdivisions of pharmacogenetics, pharmacogenomics, pharmacoepidemiology, pharmacoeconomics, and pharmacovigilance (Table 1) (1-5). There has been a quantum shift in pharmacology since the mid-1900s, with a leap from simply describing what effect a drug causes to an understanding of how drugs work.

A drug is simply a chemical or substance that causes a physiologic effect when introduced to the body (1-5). Morphine is a good example of an exogenous drug that mimics endogenous morphine (endorphins). Obviously, a wide variety of everyday chemicals (e.g., water) could be classified as a drug, and thus, drugs are generally defined on the basis of key parameters such as potency and selectivity. Importantly, some drugs elicit variable effects with varying doses - a benefit at one dose and harm at another (hormesis). The basic principle of toxicology was captured by Paracelsus in 1538 when he wrote, "sola dosis facit venenum" ("only the dose makes the poison") (6). Thallium is an example of a chemical that is used for imaging in nuclear medicine but has also been used as a poison-as depicted in old black-and-white movies and more recently in the 2015 James Bond movie Spectre-because it is highly toxic, colorless, odorless, and tasteless. A medicine is a specific chemical preparation comprising one or more drugs administered to elicit a therapeutic effect (e.g., disease or symptom treatment or prevention) (1-3). 


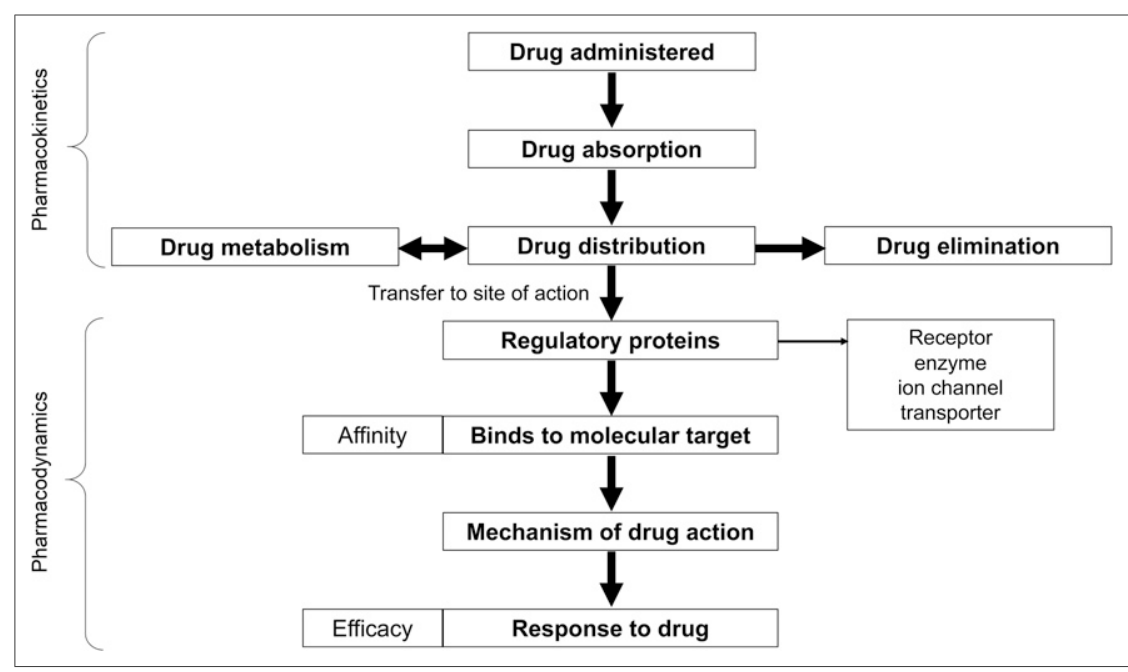

FIGURE 1. Schematic representation of relationship between pharmacokinetics and pharmacodynamics.

\section{RECEPTOR PRINCIPLE}

Receptors are proteins (macromolecules) - inside or on the surface of cells - that mediate drug activity (1-3). Receptors respond to specific neurotransmitters, hormones, antigens, chemicals, or substances. A chemical (ligand) binds to a specific site (receptor) and triggers a response (signal) in the cells $(1-3)$. The intracellular changes initiated by the ligand-receptor complex can be through direct or indirect action; however, the ligand generally functions as either an agonist or an antagonist (1-3). An agonist will mimic the endogenous ligand to produce a similar response (e.g., morphine is an agonist for opioid receptors), whereas an antagonist blocks the usual ligand and thus inhibits the physiologic response (e.g., naloxone is an antagonist for opioid receptors) (Fig. 2) (1-3). A more detailed explanation of terms regarding drug action is presented below $(1,2)$.

\section{Agonists}

An agonist creates a conformational change at the site of action that mimics the physiologic ligand. Potency is determined by affinity and efficacy. A full agonist has high efficacy. Dobutamine is an example of an adrenergic agonist.

\section{Partial Agonists}

A partial agonist demonstrates both agonist and antagonist action, which produces a truncated response. The submaximal effects represent intermediate to low efficacy. Tamoxifen is a partial-agonist drug that nuclear medicine patients might encounter. Although morphine is a full agonist for opioid receptors in the central nervous system, it is a partial agonist in other tissues such as those associated with sphincter-of-Oddi contraction.

\section{Antagonists}

An antagonist binds at the site of action but does not produce the conformational change. It does not produce a response and blocks an agonist from binding (zero efficacy). Patients in nuclear medicine departments may be taking a range of $\beta$-blockers ( $\beta$-adrenergic antagonists) and captopril, which provide angiotensin-converting-enzyme antagonism (inhibition). A competitive antagonist displaces the opportunity for the ligand or agonist to bind to the site of action.

\section{Reversible Competitive Antagonism}

Reversible competitive antagonism reflects antagonist affinity and propensity for dissociation, with a higher-affinity ligand or agonist (or indeed by virtue of higher concentration) being able to displace the antagonist. The use of aminophylline to "reverse" dipyridamole is a classic example.

\section{Irreversible Competitive Antagonism}

Irreversible competitive antagonism results when the dissociation of the antagonist from the site of action occurs either slowly or not at all. Phenoxybenzamine is an irreversible antagonist, and its use in pheochromocytoma patients for hypertension management may be seen.

\section{Inverse Agonists}

An inverse agonist produces a negative response-thus, more than simply antagonism. $\mathrm{H}_{1}$ antihistamines such as loratadine may have previously been thought of as histamine antagonists; however, they act as inverse agonists, as do several common $\mathrm{H}_{2}$ antihistamines (e.g., cimetidine and ranitidine).

\section{Allosteric Modulators}

An allosteric modulator indirectly effects action, with benzodiazepines being a typical example. They do not bind

\section{TABLE 1}

Definitions of Pharmacology Terms

Term

Pharmacodynamics

Pharmacokinetics

Pharmacogenetics

Pharmacogenomics

Pharmacoepidemiology

Pharmacoeconomics

Pharmacovigilance
Definition

Study of how drug affects living system

Study of how living system affects drug

Study of variations in drug response due to genetic influences

Study of genetic factors to guide drug therapy

Study of variability of drug response across population

Study of comparative cost-to-benefit ratios for treatment strategies Study of adverse effects of drugs 


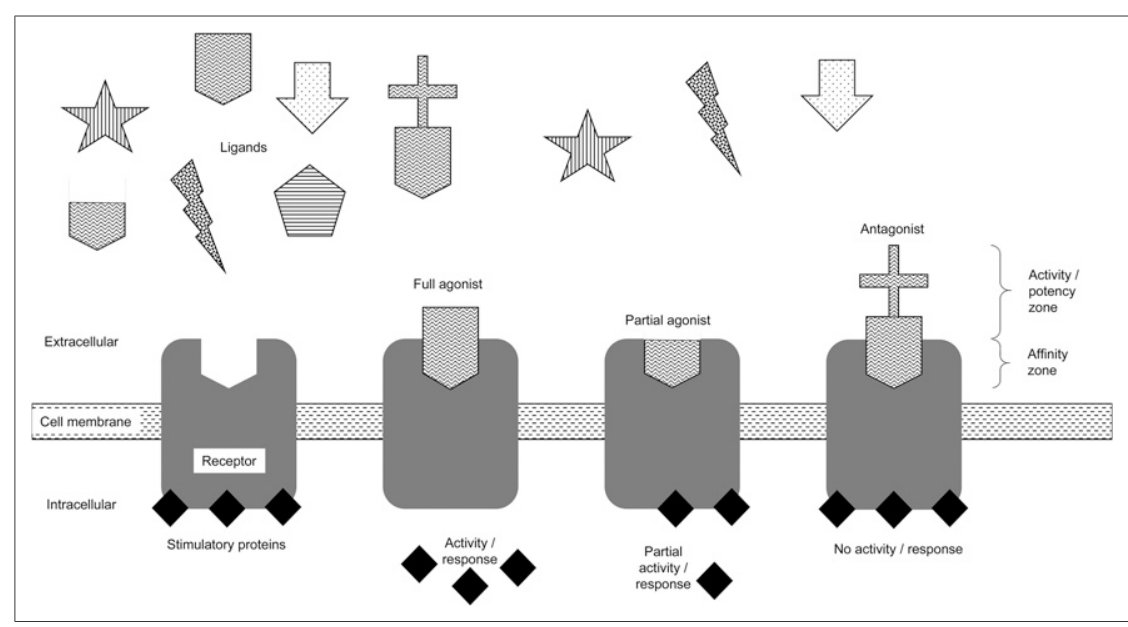

FIGURE 2. Schematic representation of receptor concept. Ligands specific for receptor may produce response across cell membrane, may produce partial response, or may block response.

at the site of action but can produce an increase (allosteric agonist) or decrease (allosteric antagonist) in the action of the ligand or agonist. Allosteric modulators may also be referred to as noncompetitive antagonists.

\section{DRUG ACTION}

Several other important terms need to be understood to characterize drugs.

\section{Specificity}

Specificity is the measure of a receptor's ability to respond to a single ligand (1-5). Low specificity generally results in physiologic responses not targeted or intended by the drug; side effects are a good example. Indeed, it is not uncommon for a drug to be developed with a theoretic action, but poor specificity for that action undermines efficacy while a side effect emerges as the new targeted role. A classic example is sildenafil (Viagra; Pfizer), which was developed to treat hypertension and angina. The drug was not particularly effective for that purpose, but an observed side effect became the new targeted role of the drug.

\section{Selectivity}

Selectivity defines the ability of the receptor to distinguish between drugs and has the same implications as specificity; indeed, the terms are often used interchangeably (1-5). Adenosine is nonselective (4 adenosine receptors with different actions) and as a result has the unwanted effect of potential bronchospasm ( $A_{1}$ receptor), whereas regadenoson is selective for the $\mathrm{A}_{2 \mathrm{~A}}$ receptor (vasodilation and bronchodilation).

\section{Affinity}

Affinity defines the strength of attraction between the drug and its receptor $(1-3,5)$. A high affinity is generally associated with a lower dose requirement (compared with low affinity for the same receptor).

\section{Potency}

Potency describes the relationship between the drug dose and the magnitude of the effect (1-5). High potency induces a strong effect with a low drug dose.

\section{Efficacy}

Efficacy is the in vivo potency, or the maximum response achieved from a drug $(1-3,5)$. The interaction (e.g., absorption, metabolism, and excretion) of the drug in the body may alter the relative bioavailability and, thus, change the theoretic effect of the drug. Rapid metabolism of a high-potency drug, for example, may render it of low efficacy whereas rapid absorption, minimal first-pass metabolism, and delayed excretion may create higher efficacy despite much lower potency.

Generally speaking, the ideal drug will be easy to administer, fully absorbed, not plasma bound, of rapid onset, spontaneously eliminated, chemically stable, highly selective and specific, and of high affinity, potency, and efficacy. It will also have a useful duration of action, a high therapeutic index (no adverse effects), and no interactions. However, there are no examples of synthetic or natural drugs that satisfy all these criteria (4). Furthermore, there may be circumstances under which these ideal properties are not wanted. For example, high affinity can also cause a prolonged action that may not be desirable, such as in the case of dipyridamole versus adenosine. High potency is also not always desirable-for example, in a drug with poor selectivity. That is, a drug that has a nonselective biodistribution will have a poorer safety profile if it is also highly potent than if it is less potent.

\section{DRUG-RECEPTOR INTERACTIONS}

Because human receptors are generally proteins, protein structure is worth reviewing. Nonetheless, receptors are not the only targets for drug binding, with other targets including ion channels, enzymes, and transporters (1). Although there are about 300 amino acids present in various animals, plants, and microbial systems, only 20 amino acids are coded by DNA to appear in proteins in humans. Cells produce proteins with different properties and activities by joining the 20 amino acids into many different combinations and sequences. The properties of proteins are determined by the physical and chemical properties of the amino acids. Proteins can be large molecules with complex 3-dimensional shapes and structures, and protein structure is best considered in terms of primary, secondary, tertiary, and quaternary structures. The primary structure relates simply to the protein configuration associated with the amino acid sequence: the order of amino acids in the polypeptide chain (peptide bonds). The secondary structure relates to the way the polypeptide chain is folded (hydrogen bonds), creating pleated sheets and helices. The tertiary structure relates to the interactions between amino acid side chains (hydrogen bonds, disulphide binds, ionic binds, and hydrophobic interactions). The quaternary structure relates to interactions between different polypeptide 


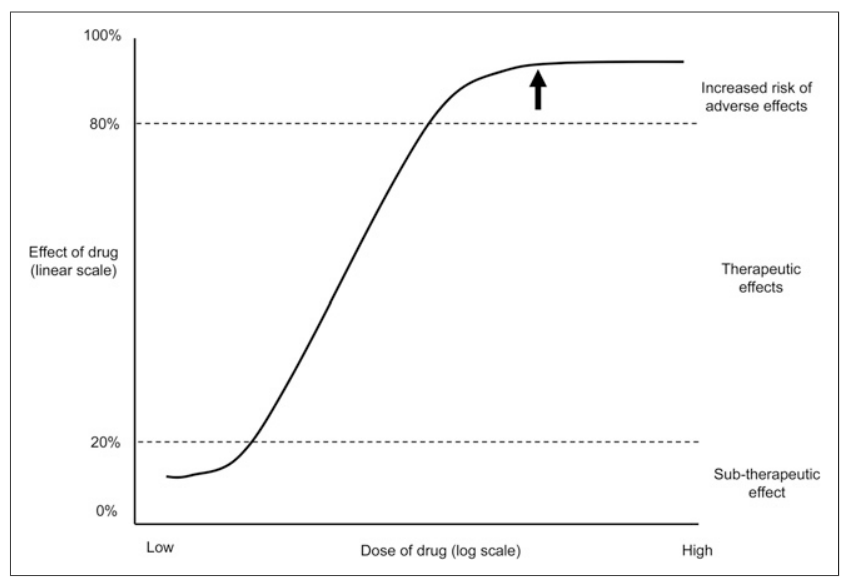

FIGURE 3. Steady-state dose-response curve. Plateau (arrow) represents dose for which maximal effect is achieved and above which additional dose does not change effect. There is also a drug dose below which no noticeable effect will be observed. Slope of line indicates how small (steep) or large (flat) a change in dose is required to observe increased effects.

chains within the same protein. Perhaps a simple way to consider protein structure would be that primary structures are an array of letters; secondary structures are creation of words from the letters; tertiary structures, a sentence from the words; and then quaternary structures, a paragraph of multiple sentences woven together. From a nuclear medicine context, $\beta$-amyloid plaque arises from a folding error (secondary structure) that results in cleavage of the protein.

All types of bonding are involved in drug-receptor interactions $(1,5)$. Although Figure 2 uses physical characteristics to schematically represent receptors, each drug-binding site has a unique chemical characteristic that is largely defined by the amino acids there $(1,5)$. How the drug and receptor interact (structure, shape, and reactivity) determines how tightly they bind. A short duration of action for a drug is generally associated with weaker bonds whereas stronger bonds produce longer-duration drug-receptor interactions (potentially irreversible). In decreasing strength, the binding forces associated with drug-receptor binding include covalent bonds, ionic interactions, hydrogen bonds, hydrophobic effects, and van der Waals forces $(1,5)$. In most cases, a combination of these interactions is involved for each drugreceptor interaction (5).

In drug-receptor binding, the rate at which the association between drug and receptor occurs relative to the rate of dissociation will define the affinity, or the strength of attraction. Low affinity, and thus higher dose requirements, is associated with drugs for which the rate of dissociation is appreciably higher than the rate of association. Conversely, high-affinity drugs requiring lower doses for effect tend to be associated with a rate of association well in excess of the rate of dissociation. The dissociation constant, which is simply the ratio of the rate of association to the rate of dissociation (smaller means higher affinity), provides insight into both the drug effect and the half-maximal effect.

\section{DOSE-RESPONSE RELATIONSHIP}

The effect of a drug is a product of the concentration of the drug at the binding site; however, specific responses to drug concentrations are typically nonlinear, and considering

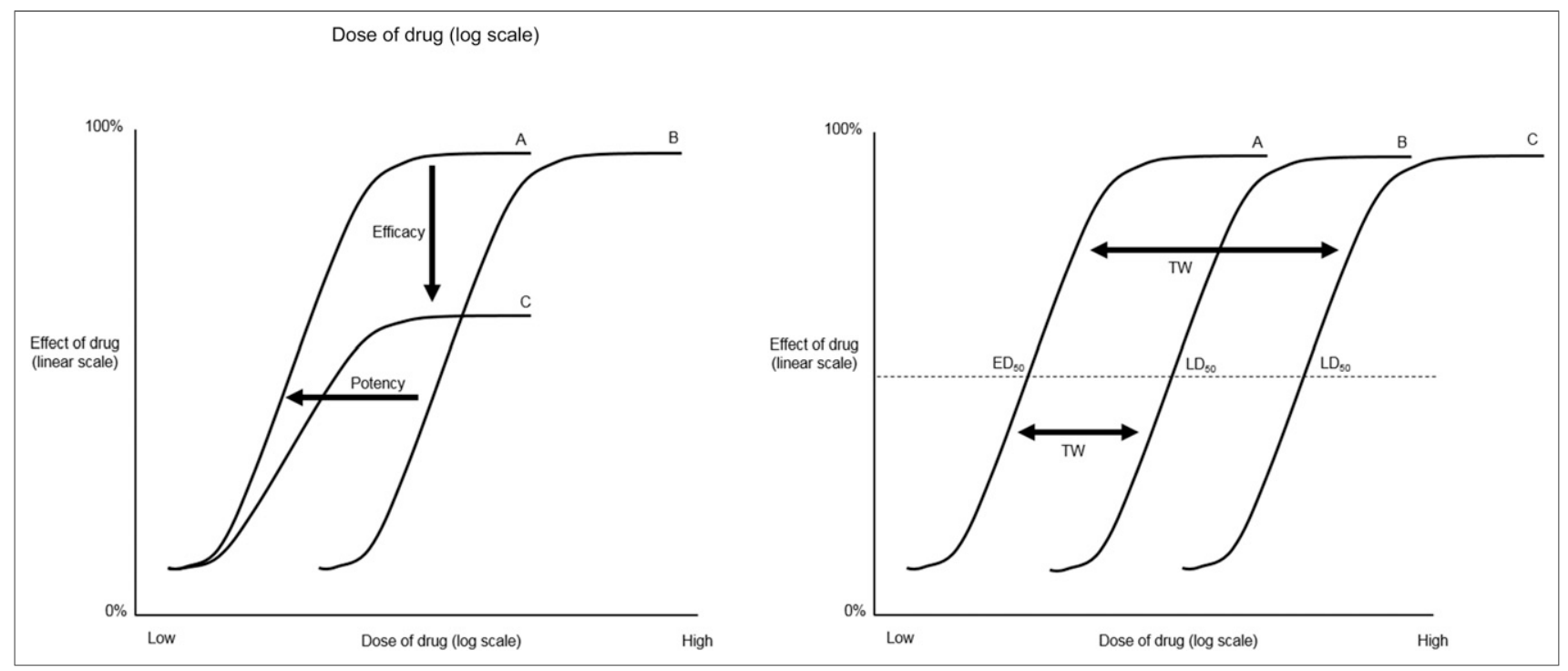

FIGURE 4. (Left) Dose-response curve representing concepts of potency and efficacy. High potency (drug A) is represented by shift to left from drug B: strong effect with low dose. Submaximal effect (drug C) despite increasing dose demonstrates lower efficacy than for drug A. (Right) Dose-response curve can also be used to represent same drug but with different outcomes (B on right). Curve $A$ represents targeted therapeutic effect, and $50 \%$ effective dose $\left(E D_{50}\right)$ is dose that produces effect in $50 \%$ of population. Curve $C$ represents same drug but more dire effects, and $50 \%$ lethal dose $\left(L D_{50}\right)$ is dose that produces death in $50 \%$ of population. Difference between curves $A$ and $C$ is therapeutic window (TW) or margin of safety and can be represented as therapeutic index by expressing ratio $\mathrm{LD}_{50} / \mathrm{ED}_{50}$. Curve $\mathrm{B}$ provides example of drug that would have narrow therapeutic window or lower therapeutic index. 


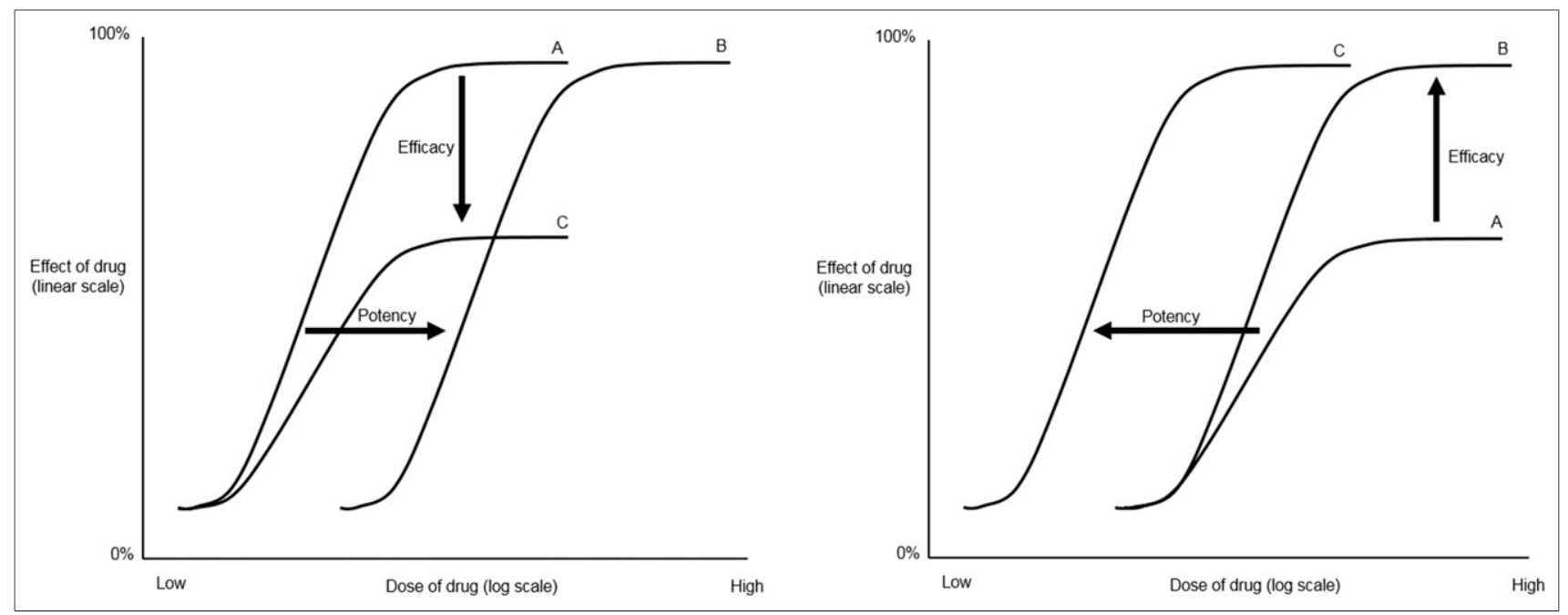

FIGURE 5. After repeated doses of drug, patient may develop tolerance (A on left), which means patient needs higher doses to generate same effect (move from curve A to B) or has lower effects from same dose (curve A to C). Individual may develop tolerance to targeted effect of drug without developing tolerance to side effects, which changes therapeutic index. After repeated doses of drug, patient may also develop sensitization (B on right), which means patient generates greater effects from same dose (move from curve A to B) or has same effects from lower dose (curve A to C). Individual may develop sensitization to one effect of drug without developing sensitization to other effects.

that drug effect is a function of dose and time, there is a significant level of complexity. To simplify this concept, dose-response curves are generated using a logarithmic $x$-axis scale for drug dose and a linear $y$-axis scale for effect at a specific time point (e.g., equilibrium/steady state, or at maximum effect) (Fig. 3). The time to steady state is influenced by several factors, including the rate of dissociation. It should be kept in mind that the drug-response curve will vary among individuals for the same drug and dose (e.g., across sex, weight, race, and age demographics), as well as within an individual (e.g., level of hydration, blood pressure, and self-limiting illness). Thus, a drug-response curve represents a mean response that can be generally applied to a population. The dose-response curve provides valuable insight into drug characteristics and allows understanding of several specific pharmacodynamic concepts $(1-3,5)$ : dose response, slope of the curve, and maximal effect (Fig. 3); potency and efficacy (Fig. 4A); 50\% effective dose, 50\% lethal dose, therapeutic window, and therapeutic index (Fig. 4B); tolerance (Fig. 5A); sensitization (Fig. 5B); and activation and antagonism (Fig. 6).

\section{DRUG INTERACTIONS}

Drug interactions can cause harm due to either increased drug effect (leading to toxicity) or decreased drug effect (leading to therapeutic failure) (1-5). There are several strategies that can be used to reduce the impact of drug interactions. The most appropriate for nuclear medicine include recognizing potential interactions between drugs (e.g., assessing patient medication history before administration of interventional medication), recognizing factors that might potentiate drug interaction (e.g., age, diet, hydration level, comorbidity, and environmental factors), and recognizing drugs with a narrow therapeutic index.

Interactions between drugs (drug-drug interactions) are an important cause of patient harm and are particularly important to consider in nuclear medicine patients because their mean age exceeds $60 \mathrm{y}$ and both polypharmacy (concurrent use of multiple medications) and the degenerative effects of aging on organ function increase the risk of interactions. Pharmacokinetic drug-drug interactions will be discussed in part 2 of this series of articles. The effects of such interactions can be cumulative (e.g., angiotensin-converting-enzyme [ACE] inhibitors act on

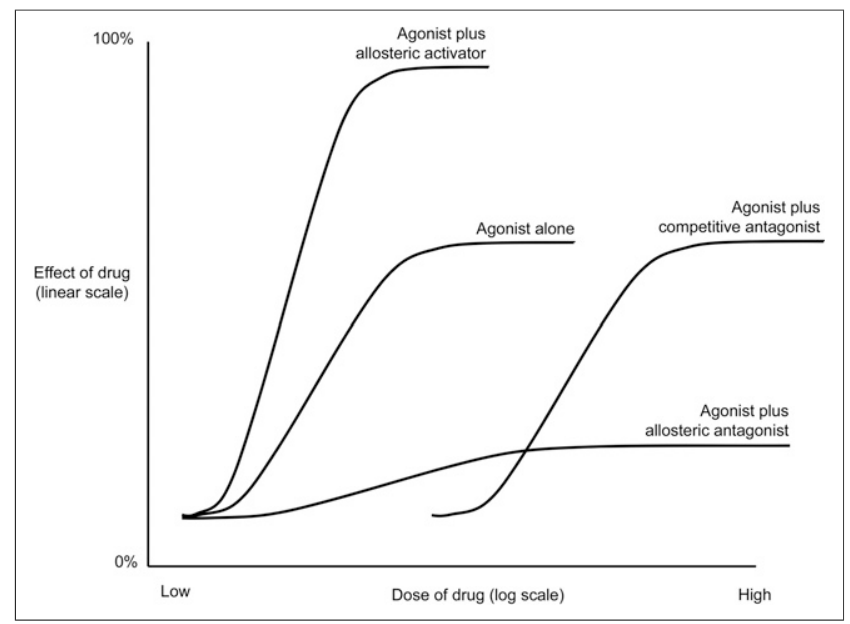

FIGURE 6. Agonist activity at receptor may be altered by antagonist, potentiated by allosteric activation of other receptors, or inhibited by allosteric inhibition at other receptors. 


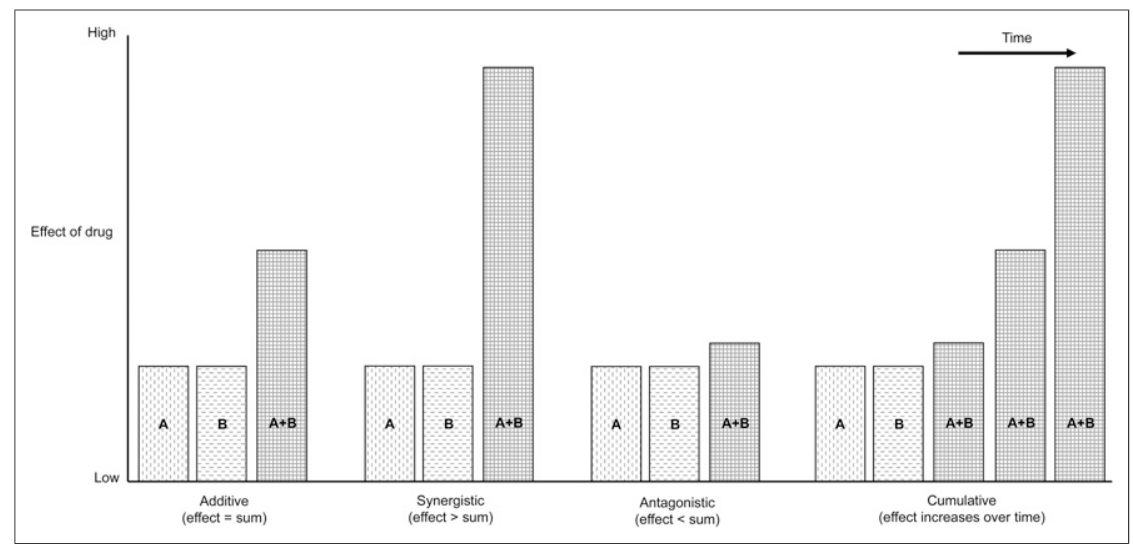

FIGURE 7. Schematic representation of pharmacodynamic drug-drug interactions. body. Nonetheless, pharmacodynamics provides essential insights into the effects of interventional and adjunctive medications on the nuclear medicine patient. Furthermore, understanding of pharmacodynamic principles provides the tools to mitigate interactions between medications that are prescribed adjunctively or interventionally and medications that are prescribed on a recurring basis. This article provides a foundational understanding for a more detailed examination of adjunctive and interventional medications in subsequent articles in this series. potassium-sparing diuretics to lead to hyperkalemia), additive (e.g., ACE inhibitors and loop diuretics reduce blood pressure), synergistic (e.g., alcohol and sedatives have an effect greater than the sum of the individual drugs), or antagonistic (e.g., nonsteroidal antiinflammatory drugs reduce the effect of ACE inhibitors on blood pressure) (Fig. 7).

\section{CONCLUSION}

Although dose-response curves apply some translational principles to therapeutic nuclear medicine, the tracer principle associated with radiopharmaceuticals generally relegates pharmacodynamics to the periphery. Pharmacodynamics is the study of how the drug affects the body, and by design, radiopharmaceuticals should have little or no effect on the

\section{DISCLOSURE}

No potential conflict of interest relevant to this article was reported.

\section{REFERENCES}

1. Rang H, Dale M, Ritter J, Flower R. Rang and Dale's Pharmacology. 6th ed. London, U.K.: Churchill Livingston; 2008:3-112.

2. Waller D, Renwick A, Hillier K. Medical Pharmacology and Therapeutics. 2nd ed. London, U.K.: Elsevier; 2006:3-85.

3. Bryant B, Knights K, Salerno E. Pharmacology for Health Professionals. 2nd ed. Sydney, Australia: Mosby Elsevier; 2007:1-215.

4. Greenstein B. Rapid Revision in Clinical Pharmacology. New York, NY: Radcliffe Publishing; 2008:4-19.

5. Golan DE, Tashjian AH, Armstrong EJ, Armstrong AW. Principles of Pharmacology: The Pathophysiologic Basis of Drug Therapy. 3rd ed. Philadelphia, PA: Lippincott, Williams \& Wilkins; 2012:1-79.

6. Stumpf WE. The dose makes the medicine. Drug Discov Today. 2006;11:550-1555. 